\title{
A COMPLEX STRUCTURE ON THE SET OF QUASICONFORMALLY EXTENDIBLE NON-OVERLAPPING MAPPINGS INTO A RIEMANN SURFACE
}

\author{
DAVID RADNELL AND ERIC SCHIPPERS
}

\begin{abstract}
Let $\Sigma$ be a Riemann surface with $n$ distinguished points $p_{1}, \ldots, p_{n}$. We prove that the set of $n$-tuples $\left(\phi_{1}, \ldots, \phi_{n}\right)$ of univalent mappings $\phi_{i}$ from the unit disc $\mathbb{D}$ into $\Sigma$ mapping 0 to $p_{i}$, with non-overlapping images and quasiconformal extensions to a neighbourhood of $\overline{\mathbb{D}}$, carries a natural complex Banach manifold structure. This complex structure is locally modelled on the $n$-fold product of a two complex-dimensional extension of the universal Teichmüller space. Our results are motivated by Teichmüller theory and two-dimensional conformal field theory.
\end{abstract}

\section{INTRODUCTION}

Let $\overline{\mathbb{C}}$ be the Riemann sphere, $\mathbb{D}=\{z \in \mathbb{C}|| z \mid<1\}$ and $\mathbb{D}^{*}=\{z \in \overline{\mathbb{C}}|| z \mid>1\}$.

Definition 1.1 (of $\mathcal{O}_{q c}(\Sigma)$ ). Let $\Sigma$ be a Riemann surface with $n$ distinguished points $p_{1}, \ldots, p_{n}$. Define $\mathcal{O}_{\mathrm{qc}}(\Sigma)$ to be the set of $n$-tuples $\left(\phi_{1}, \ldots, \phi_{n}\right)$ where $\phi_{i}: \mathbb{D} \rightarrow \Sigma$ are maps with the following properties:

(1) $\phi_{i}(0)=p_{i}$

(2) $\phi_{i}$ is one-to-one and holomorphic on $\mathbb{D}$

(3) $\phi_{i}$ has a quasiconformal extension to an open neighbourhood of $\overline{\mathbb{D}}$

(4) $\phi_{i}(\overline{\mathbb{D}}) \cap \phi_{j}(\overline{\mathbb{D}})=\emptyset$ whenever $i \neq j$.

Remark 1.2. The space $\mathcal{O}_{q c}(\Sigma)$ was introduced by the authors in [9, Definition 5.1]. In this paper the direction of the maps has been reversed and the notation slightly modified.

Remark 1.3. The motivation for this work came from the case of compact $\Sigma$. The authors thank the referee for carefully reading the manuscript and for pointing out that our results hold when $\Sigma$ is an arbitrary Riemann surface.

Definition 1.4. The model space $\mathcal{O}_{\mathrm{qc}}$ is defined as follows:

$$
\begin{aligned}
\mathcal{O}_{\mathrm{qc}}=\{f: \mathbb{D} \rightarrow \mathbb{C} \mid f \text { is one-to-one, holomorphic, has quasiconformal extension to } \mathbb{C}, \\
\text { and } f(0)=0\} .
\end{aligned}
$$

Our main result is that $\mathcal{O}_{\mathrm{qc}}(\Sigma)$ is a complex Banach manifold with complex structure locally modelled on $\mathcal{O}_{\mathrm{qc}} \times \cdots \times \mathcal{O}_{\mathrm{qc}}$ (Theorem 3.11).

Date: August 22, 2021.

2000 Mathematics Subject Classification. 30C55, 30C62, 30F60, 81T40.

Key words and phrases. Teichmüller spaces, quasiconformal extensions, univalent maps, rigged moduli spaces, conformal field theory. 
Non-overlapping univalent mappings are a natural object of study in geometric function theory and have been extensively researched, especially in connection with extremal problems and inequalities. If $\Sigma=\overline{\mathbb{C}}$ with $n$ distinguished points, then the class $\mathcal{O}_{\mathrm{qc}}(\Sigma)$ with the restriction that the maps have quasiconformal extension removed is referred to as the Goluzin-Lebedev class.

Non-overlapping maps with quasiconformal extension have also been studied a great deal, especially in the case of pairs of non-overlapping maps. The condition that the maps have quasiconformal extension usually results in sharpened inequalities. A recent review of the literature on non-overlapping maps can be found in [4].

In the case of several variables, Forstnerič [3] showed that the set of holomorphic mappings of pseudoconvex domains into a complex manifold possesses a complex structure, for various choices of regularity of the mappings on the boundary.

In this paper we consider the problem of the existence of a complex structure on $\mathcal{O}_{\mathrm{qc}}(\Sigma)$. For compact $\Sigma$, this question is motivated by both Teichmüller theory and conformal field theory. In conformal field theory, a fundamental object is the rigged moduli space. This infinite-dimensional moduli space is the space of conformal equivalence classes of Riemann surfaces together with specified local coordinates at the $n$ distinguished points (see [9] for details and references). In [9] we showed, by generalizing to elements of $\mathcal{O}_{\mathrm{qc}}(\Sigma)$ as the local coordinate data at the distinguished points, that this moduli space has a natural complex structure inherited from the infinite-dimensional Teichmüller space of Riemann surfaces with $n$ boundary curves. Furthermore, our results in [9] suggested that the Teichmüller space of genus $g$ Riemann surfaces with $n$ boundary curves is fibred over the (finite-dimensional) Teichmüller space of $n$-punctured, genus $g$ surfaces. The fibres are $\mathcal{O}_{\text {qc }}(\Sigma)$, so these fibres should inherit a complex structure.

In this paper we show that $\mathcal{O}_{\mathrm{qc}}(\Sigma)$ possesses a natural complex structure arising from a different point of view. We construct this complex structure from a two-complex-dimensional extension of the universal Teichmüller space using recent results of Teo [10]. Interestingly, our construction does not have any apparent connection with the fact that the Teichmüller space of a Riemann surface with $n$ boundary curves is contained in the universal Teichmüller space. Nevertheless, we suspect that these two complex structures are compatible, and we hope to settle the question in a future publication.

\section{Preliminaries}

In this section we summarize some known results about the Bers embedding which will be needed in the rest of the paper. Some of these results are standard, but we also rely on results of Teo [10]. Details can be found in [1], [5], [8] and [10].

Using the normalizations in [10] and [1] we define the following function spaces:

$$
\begin{gathered}
\mathcal{D}=\{f: \mathbb{D} \rightarrow \overline{\mathbb{C}} \mid f \text { is one-to-one, holomorphic, has quasiconformal extension to } \overline{\mathbb{C}}, \\
\text { and } \left.f(0)=0, f^{\prime}(0)=1, f^{\prime \prime}(0)=0\right\}
\end{gathered}
$$

and

$$
\begin{gathered}
\widetilde{\mathcal{D}}=\{f: \mathbb{D} \rightarrow \mathbb{C} \mid f \text { is one-to-one, holomorphic, has quasiconformal extension to } \mathbb{C}, \\
\text { and } \left.f(0)=0, f^{\prime}(0)=1\right\} .
\end{gathered}
$$


The function space $\mathcal{D}$ is one of several standard models of the universal Teichmüller space. Let $L_{1}^{\infty}\left(\mathbb{D}^{*}\right)$ denote the set of measurable functions on $\mathbb{D}^{*}$ with sup norm strictly less than one. We define an equivalence relation on $L_{1}^{\infty}\left(\mathbb{D}^{*}\right)$ as follows: $\mu \sim \nu$ if and only if the normalized solutions $w_{\mu}: \mathbb{D}^{*} \rightarrow \mathbb{D}^{*}$ and $w_{\nu}: \mathbb{D}^{*} \rightarrow \mathbb{D}^{*}$ to the Beltrami equation satisfy $w_{\mu}=w_{\nu}$ on $\partial \mathbb{D}^{*}$. We then define the universal Teichmüller space

$$
T\left(\mathbb{D}^{*}\right)=L_{1}^{\infty}\left(\mathbb{D}^{*}\right) / \sim .
$$

Furthermore, $\mu \sim \nu$ if and only if the solutions $f_{\mu}$ and $f_{\nu}$ to the Beltrami equation on $\overline{\mathbb{C}}$ with $\mu$ and $\nu$ extended to be 0 on $\mathbb{D}$ (normalized so that $f(0)=f^{\prime}(0)-1=f^{\prime \prime}(0)=0$ ) satisfy $f_{\mu}=f_{\nu}$ on $\mathbb{D}$. Thus, since their restrictions to $\mathbb{D}$ are holomorphic and one-to-one, $T\left(\mathbb{D}^{*}\right)$ is in one-to-one correspondence with $\mathcal{D}$.

Remark 2.1. The normalization for $\mathcal{D}$ is not the usual one, but it is convenient in our setting.

Remark 2.2. In [10] and [11], the space $\mathcal{D}$ is defined slightly differently: functions in $\mathcal{D}$ have range confined to $\mathbb{C}$. This seems to be a minor oversight, since if $\mathcal{D}$ is to be identified with $T\left(\mathbb{D}^{*}\right)$, then the normalization $f^{\prime \prime}(0)=0$ cannot be imposed without allowing maps in $\mathcal{D}$ to take the value $\infty$.

The space $\widetilde{\mathcal{D}}$ is a model of the universal Teichmüller curve $\mathcal{T}\left(\mathbb{D}^{*}\right)$ of Bers [1, 10, 11]. Although the identification of $T\left(\mathbb{D}^{*}\right)$ with $\mathcal{D}$ will be directly used in this paper, the identification of $\mathcal{T}\left(\mathbb{D}^{*}\right)$ with $\widetilde{\mathcal{D}}$ will not be. However, some of the arguments in this paper regarding the complex structures of the spaces could equally well be phrased in terms of $\mathcal{T}\left(\mathbb{D}^{*}\right)$ rather than $T\left(\mathbb{D}^{*}\right)$.

Remark 2.3. By conformal welding one can make the identifications

$$
\mathcal{D} \cong T\left(\mathbb{D}^{*}\right) \cong \mathrm{QS}\left(S^{1}\right) / \operatorname{Möb}\left(S^{1}\right)
$$

and

$$
\widetilde{\mathcal{D}} \cong \mathcal{T}\left(\mathbb{D}^{*}\right) \cong \mathrm{QS}\left(S^{1}\right) / \operatorname{Rot}\left(S^{1}\right)
$$

where $Q S\left(S^{1}\right)$ denotes the set of quasisymmetric maps from the unit circle $|z|=1$ to itself, and $\operatorname{Rot}\left(S^{1}\right)$ is the set of transformations $z \mapsto e^{i \theta} z$. In the Appendix (Section 4) it is shown that there is a natural bijection

$$
\mathcal{O}_{\mathrm{qc}} \cong \mathrm{QS}\left(S^{1}\right) \times \mathbb{R}
$$

which can be seen as the next step in this progression of removing normalizations from $T\left(\mathbb{D}^{*}\right)$. This fact is not used in the rest of the paper, but is an interesting point of interpretation.

We briefly outline how $\mathcal{D}$ and $\widetilde{\mathcal{D}}$ can be mapped respectively into open subsets of the Banach spaces

$$
A_{\infty}^{2}(\mathbb{D})=\left\{\psi(z): \mathbb{D} \rightarrow \mathbb{C} \mid \psi \text { holomorphic, }\|\psi\|_{2, \infty}=\sup _{z \in \mathbb{D}}\left(1-|z|^{2}\right)^{2}|\psi(z)|<\infty\right\}
$$

and

$$
A_{\infty}^{1}(\mathbb{D})=\left\{\phi(z): \mathbb{D} \rightarrow \mathbb{C} \mid \phi \text { holomorphic, }\|\phi\|_{1, \infty}=\sup _{z \in \mathbb{D}}\left(1-|z|^{2}\right)|\phi(z)|<\infty\right\}
$$


Remark 2.4. $A_{\infty}^{2}(\mathbb{D})$ should be interpreted as a space of holomorphic quadratic differentials $\psi(z) d z^{2}$, and $A_{\infty}^{1}(\mathbb{D})$ should be interpreted as a space of first-order holomorphic differentials $\phi(z) d z$.

We define two differential operations on holomorphic functions

$$
\mathcal{A}(f)=\frac{f^{\prime \prime}}{f^{\prime}}
$$

and

$$
\mathcal{S}(f)=\frac{f^{\prime \prime \prime}}{f^{\prime}}-\frac{3}{2}\left(\frac{f^{\prime \prime}}{f^{\prime}}\right)^{2}
$$

which are called the "pre-Schwarzian derivative" and the "Schwarzian derivative" respectively. The Bers embedding $\mathcal{S}: \mathcal{D} \rightarrow A_{\infty}^{2}(\mathbb{D})$ maps the universal Teichmüller space injectively into the Banach space $A_{\infty}^{2}(\mathbb{D})$. A well-known result of Ahlfors says that the image is open, and thus $T\left(\mathbb{D}^{*}\right)$ and $\mathcal{D}$ inherit a complex structure. This complex structure is compatible with that inherited from $L_{1}^{\infty}\left(\mathbb{D}^{*}\right)$ by results of Bers [1].

We now define the natural injections of $\widetilde{\mathcal{D}}$ into the Banach spaces $A_{\infty}^{1}(\mathbb{D})$ and $A_{\infty}^{2}(\mathbb{D}) \oplus \mathbb{C}$. Firstly, there is the "Bers embedding" of the universal Teichmüller curve:

$$
\begin{aligned}
\beta: \widetilde{\mathcal{D}} & \rightarrow A_{\infty}^{2}(\mathbb{D}) \oplus \mathbb{C} \\
f & \mapsto\left(\mathcal{S}(f), \frac{1}{2} \mathcal{A}(f)(0)\right) .
\end{aligned}
$$

Bers showed that the image of $\beta$ is open [1]. Secondly, there is the injection defined by

$$
\begin{aligned}
\hat{\beta}: \widetilde{\mathcal{D}} & \rightarrow A_{\infty}^{1}(\mathbb{D}) \\
f & \mapsto \mathcal{A}(f) .
\end{aligned}
$$

Using these injections we define metrics on $\mathcal{D}$ and $\widetilde{\mathcal{D}}$ as follows. For $f_{1}, f_{2} \in \mathcal{D}$, let

$$
d_{s}\left(f_{1}, f_{2}\right)=\left\|\mathcal{S}\left(f_{1}\right)-\mathcal{S}\left(f_{2}\right)\right\|_{2, \infty} .
$$

For $f_{1}, f_{2} \in \widetilde{\mathcal{D}}$, let

$$
d_{p s}\left(f_{1}, f_{2}\right)=\left\|\mathcal{A}\left(f_{1}\right)-\mathcal{A}\left(f_{2}\right)\right\|_{1, \infty} .
$$

Remark 2.5. By the invariance of the Schwarzian under post-composition by Möbius transformations, $\mathcal{S}(\widetilde{\mathcal{D}})=\mathcal{S}(\mathcal{D})$. In particular $\mathcal{S}(\widetilde{\mathcal{D}})$ is open in $A_{\infty}^{2}(\mathbb{D})$.

We require results of Teo [10] concerning the compatibility of the complex structures on $\widetilde{\mathcal{D}}$ induced from $A_{\infty}^{1}(\mathbb{D})$ and $A_{\infty}^{2}(\mathbb{D}) \oplus \mathbb{C}$ by $\hat{\beta}$ and $\beta$ respectively. These are summarized in the following four theorems.

Define maps

$$
\begin{aligned}
\Psi: A_{\infty}^{1}(\mathbb{D}) & \rightarrow A_{\infty}^{2}(\mathbb{D}) \\
g & \mapsto g^{\prime}-\frac{g^{2}}{2}
\end{aligned}
$$


and

$$
\begin{aligned}
\hat{\Psi}: A_{\infty}^{1}(\mathbb{D}) & \rightarrow A_{\infty}^{2}(\mathbb{D}) \oplus \mathbb{C} \\
g & \mapsto\left(\Psi(g), \frac{1}{2} g(0)\right) .
\end{aligned}
$$

The importance of these maps stems from the identities $\Psi \circ \mathcal{A}=\mathcal{S}$ and $\hat{\Psi} \circ \hat{\beta}=\beta$.

Theorem 2.6. [10, Corollary A.3] $\Psi$ is holomorphic.

Theorem 2.7. [10, Theorem A.5] $\hat{\Psi}$ is one-to-one and holomorphic.

Theorem 2.8. The map $\hat{\beta}$ is one-to-one and the image is open in $A_{\infty}^{1}(\mathbb{D})$. In particular $\widetilde{\mathcal{D}}$ inherits a complex structure from $A_{\infty}^{1}(\mathbb{D})$.

Proof. $\hat{\beta}$ is clearly one-to-one, since if $\mathcal{A}(f)=\mathcal{A}(g)$ we have that in some open set

$$
\log f^{\prime}=\log g^{\prime}+C
$$

for some branch of logarithm and constant $C$ (note $f^{\prime} \neq 0$ and $g^{\prime} \neq 0$ since they are univalent), so $f^{\prime}=e^{C} g^{\prime}$. Since $f^{\prime}(0)=g^{\prime}(0)$ and $f(0)=g(0)=0$ it follows that $f=g$.

The image of $\beta$ is open in $A_{\infty}^{2}(\mathbb{D}) \oplus \mathbb{C}$ by a result of Bers [1], as mentioned above. Since $\hat{\Psi} \circ \hat{\beta}=\beta$ and $\hat{\Psi}$ is continuous and one-to-one, this implies that the image of $\hat{\beta}$ is open.

Theorem 2.9. The restriction $\hat{\Psi}: \hat{\beta}(\widetilde{\mathcal{D}}) \rightarrow \beta(\widetilde{\mathcal{D}})$ is a biholomorphism.

Proof. This is stated and proved in the proof of [10, Theorem A.6].

Remark 2.10. In particular, $A_{\infty}^{1}(\mathbb{D})$ and $A_{\infty}^{2}(\mathbb{D})$ induce the same complex structure on $\widetilde{\mathcal{D}}$ via $\hat{\beta}$ and $\beta$ respectively. This is the actual statement of [10, Theorem A.6].

Remark 2.11. Teo's Theorem 2.9 is not an immediate consequence of Theorems 2.7 and 2.8. One-to-one holomorphic maps in infinite dimensions do not necessarily have holomorphic inverses.

\section{Complex structure on $\mathcal{O}_{\text {qc }}$ And $\mathcal{O}_{\text {qc }}(\Sigma)$}

3.1. Complex structure on $\mathcal{O}_{\mathrm{qc}}$. In the rest of the paper, we will always consider $\mathcal{D}$ and $\widetilde{\mathcal{D}}$ to have the topology induced by the metrics $d_{s}$ and $d_{p s}$.

We now define an embedding of $\mathcal{O}_{q c}$ into a Banach space:

$$
\begin{aligned}
\chi: \mathcal{O}_{q c} & \rightarrow A_{\infty}^{1}(\mathbb{D}) \oplus \mathbb{C} \\
f & \mapsto\left(\mathcal{A}(f), f^{\prime}(0)\right) .
\end{aligned}
$$

The Banach space direct sum norm on $A_{\infty}^{1}(\mathbb{D}) \oplus \mathbb{C}$ is defined by $\|(\phi, c)\|=\|\phi\|_{1, \infty}+|c|$.

Theorem 3.1. $\chi$ is one-to-one, and the image is $\hat{\beta}(\widetilde{\mathcal{D}}) \oplus \mathbb{C}^{*}$. In particular, $A_{\infty}^{1} \oplus \mathbb{C}$ induces a complex Banach manifold structure on $\mathcal{O}_{\mathrm{qc}}$ via $\chi$.

Proof. Assume that $\chi(f)=\chi(g)$ : then $f^{\prime \prime} / f^{\prime}=g^{\prime \prime} / g^{\prime}, g^{\prime}(0)=f^{\prime}(0)$ and $f(0)=g(0)=0$. Thus the same reasoning as in the proof of Theorem 2.8 implies that $f=g$.

Since $f \in \mathcal{O}_{q c}$ implies $\alpha f \in \mathcal{O}_{\mathrm{qc}}$ for any $\alpha \in \mathbb{C}^{*}$ and $\mathcal{A}(\alpha f)=\mathcal{A}(f)$, if $(\psi, a) \in \chi\left(\mathcal{O}_{\mathrm{qc}}\right)$ then so is $(\psi, \alpha a)$ for all $\alpha \in \mathbb{C}^{*}$. Furthermore $\mathcal{A}\left(\mathcal{O}_{\text {qc }}\right)=\mathcal{A}(\widetilde{\mathcal{D}})=\hat{\beta}(\widetilde{\mathcal{D}})$. It follows that $\chi\left(\mathcal{O}_{\mathrm{qc}}\right)=\hat{\beta}(\widetilde{\mathcal{D}}) \oplus \mathbb{C}^{*}$. 
The last claim follows from the fact that $\hat{\beta}(\widetilde{\mathcal{D}})$ is open by Theorem 2.8 .

The metric on $A_{\infty}^{1}(\mathbb{D}) \oplus \mathbb{C}$ induces the metric

$$
d_{o}(f, g)=\|\chi(f)-\chi(g)\|=\|\mathcal{A}(f)-\mathcal{A}(g)\|_{1, \infty}+\left|f^{\prime}(0)-g^{\prime}(0)\right|
$$

on $\mathcal{O}_{\text {qc }}$. With the topology induced by this metric we immediately have

Proposition 3.2. The map

$$
\begin{aligned}
\mathcal{O}_{\mathrm{qc}} & \rightarrow \widetilde{\mathcal{D}} \\
f & \mapsto f / f^{\prime}(0)
\end{aligned}
$$

is continuous.

A few results about the topology of $\mathcal{O}_{\mathrm{qc}}$ will be necessary. We will need the fact that point evaluation at $z$ is a continuous map on $\widetilde{\mathcal{D}}$ and $\mathcal{O}_{\mathrm{qc}}$ for any $z \in \overline{\mathbb{D}}$. We will also need a theorem to the effect that if $f$ and $g$ are nearby in $\widetilde{\mathcal{D}}$ then $f\left(S^{1}\right)$ is uniformly close to $g\left(S^{1}\right)$, and similarly for $\mathcal{O}_{\mathrm{qc}}$.

In order to do this we will make use of the Teichmüller metric, which is defined as follows. Let $f$ and $g$ be elements of $\mathcal{D}$, where $\mathcal{D}$ is considered as a model for $T\left(\mathbb{D}^{*}\right)$. The Teichmüller distance between $f$ and $g$ is defined to be

$$
\tau(f, g)=\frac{1}{2} \inf _{\mu, \nu}\left(\frac{1+\|(\mu-\nu) /(1-\bar{\mu} \nu)\|_{\infty}}{1-\|(\mu-\nu) /(1-\bar{\mu} \nu)\|_{\infty}}\right)
$$

where $\mu$ and $\nu$ are the dilatations of quasiconformal extensions of $f$ and $g$ respectively, and the minimum is taken over all such choices of extension. The Teichmüller metric is compatible with the Schwarzian metric $d_{s}$ introduced in Section 2 (see [5, III.4.2, III.4.3]). Furthermore, if one fixes a quasiconformal extension $\tilde{f}$ of $f$ to $\overline{\mathbb{C}}$ with dilatation $\mu_{0}$, and takes the minimum over $\nu$ only, then the result is the same (see [5, Lemma III.2.1]). That is,

$$
\tau(f, g)=\frac{1}{2} \inf _{\nu}\left(\frac{1+\left\|\left(\mu_{0}-\nu\right) /\left(1-\overline{\mu_{0}} \nu\right)\right\|_{\infty}}{1-\left\|\left(\mu_{0}-\nu\right) /\left(1-\overline{\mu_{0}} \nu\right)\right\|_{\infty}}\right) .
$$

Note that, if $K_{h}$ denotes the maximal dilatation of a quasiconformal map $h$, then

$$
\tau(f, g)=\frac{1}{2} \inf _{\tilde{g}} \log K_{\tilde{g} \circ \tilde{f}^{-1}} .
$$

where $\tilde{f}$ is a fixed quasiconformal extension of $f$ and $\tilde{g}$ are quasiconformal extensions of $g$ to $\overline{\mathbb{C}}$.

Theorem 3.3. The point evaluation map $f \mapsto f(z)$ is continuous on $\widetilde{\mathcal{D}}$ for any $z \in \overline{\mathbb{D}}$. Furthermore, for any $f \in \widetilde{\mathcal{D}}$ and $\epsilon>0$, there is an open neighbourhood $U \subset \widetilde{\mathcal{D}}$ containing $f$ such that for any $g \in U$ and $y \in \partial \mathbb{D},|g(y)-f(y)|<\epsilon$.

Proof. First, fix any $K>1$ and $R>1$. Consider the family of K-quasiconformal mappings of the disc $|z|<R$ whose restriction to $\mathbb{D}$ is in $\widetilde{\mathcal{D}}$. This family is equicontinuous in $|z|<R$ by [6. Theorem 4.2], since the family omits $\infty$, and for any point $z$ such that $|z|<1, f(z)$ is uniformly bounded away from $\infty$ by the growth theorem for univalent mappings of the disc.

Now fix $f \in \widetilde{\mathcal{D}}$. Fix a quasiconformal extension $\tilde{f}$ of $f$, and let $K>1$ be the maximal dilatation of $f$. Finally fix a $K_{1}>K$. We claim that there is a $\delta_{1}>0$ such that if 
$d(f, g)_{p s}<\delta_{1}$ then $g$ has an extension with maximal dilatation less than $K_{1}$. To see this, choose Möbius transformations $T_{1}$ and $T_{2}$ such that $T_{1} \circ f, T_{2} \circ g \in \mathcal{D}$. By the compatibility of the Schwarzian metric $d_{s}$ on $\mathcal{D}$ and the Teichmüller metric $\tau$ in the form (3.1), there is a $\delta_{2}$ such that $d_{s}\left(T_{1} \circ f, T_{2} \circ g\right)<\delta_{2}$ implies that $T_{2} \circ g$ has a quasiconformal extension to $\overline{\mathbb{C}}$ with maximal dilatation less than or equal to $K_{1}$. Since for any Möbius transformation $T$, post-composition by $T$ does not affect the maximal dilatation of a quasiconformal map, and since $\mathcal{S}(T \circ f)=\mathcal{S}(f)$, it follows that if $\|\mathcal{S}(f)-\mathcal{S}(g)\|_{1, \infty}<\delta_{2}$ then $g$ has a quasiconformal extension of dilatation less than $K_{1}$. Since $\Psi$ is continuous by Theorem 2.6, and $\Psi \circ \mathcal{A}=\mathcal{S}$, the claim follows.

We now show that point evaluation is continuous in $\widetilde{\mathcal{D}}$ for $z \in \overline{\mathbb{D}}$. First observe that the claim is immediate for $z \in \mathbb{D}$ since convergence in $d_{p s}$ implies uniform convergence in compact subsets of $\mathbb{D}$. To prove the claim for $x \in S^{1}$, fix $f \in \widetilde{\mathcal{D}}$ and a quasiconformal extension $\tilde{f}$; this $\tilde{f}$ has maximal dilatation $K$ for some $K>1$. For $K_{1}>K$, recall that the family $\mathcal{F}$ of mappings in $\widetilde{\mathcal{D}}$ with quasiconformal extensions to $|z|<R$ of maximal dilatation less than $K_{1}$ is equicontinuous in $\overline{\mathbb{D}}$ by the first paragraph of the proof. This family includes $f$ itself. Furthermore, the boundary values of $g \in \mathcal{F}$ do not depend on the choice of extension. We show that point evaluation $g \mapsto g(x)$ is continuous at $f$. Choose a $y \in \mathbb{D}$ such that $|g(x)-g(y)|<\epsilon / 3$ for any $g \in \mathcal{F}$; this can be done by the equicontinuity of $\mathcal{F}$. There is a $\delta$ such that $d_{p s}(f, g)<\delta \Rightarrow|f(y)-g(y)|<\epsilon / 3$. Shrinking $\delta$ if necessary, by the previous paragraph we may take $\delta$ so that $d_{p s}(f, g)<\delta$ implies that $g \in \mathcal{F}$. Thus $|g(x)-f(x)| \leq|g(x)-g(y)|+|g(y)-f(y)|+|f(y)-f(x)|<\epsilon$.

Since $\partial \mathbb{D}$ is compact, to prove the last claim of the Theorem it suffices to show that for any $x \in \partial \mathbb{D}$ and $\epsilon>0$, there is an open subinterval of $\partial \mathbb{D}$ containing $x$ and a $\delta$ such that $d_{p s}(f, g)<\delta \Rightarrow|g(y)-f(y)|<\epsilon$ for all $y$ in the interval. By the equicontinuity of $\mathcal{F}$ there is an open subinterval $I$ of $\partial \mathbb{D}$ containing $x$ and a $\delta$ such that for all $y \in I$, if $g$ satisfies $d_{p s}(f, g)<\delta$ then $|g(y)-g(x)|<\epsilon / 3$. Furthermore by the continuity of point evaluations, by shrinking $\delta$ if necessary we can ensure that $|g(x)-f(x)|<\epsilon / 3$. Thus another three- $\epsilon$ argument

$$
|g(y)-f(y)| \leq|g(y)-g(x)|+|g(x)-f(x)|+|f(x)-f(y)|<\epsilon
$$

completes the proof.

Corollary 3.4. The point evaluation map $f \mapsto f(z)$ is continuous on $\mathcal{O}_{\mathrm{qc}}$ for any $z \in \overline{\mathbb{D}}$. Furthermore, for any $f \in \mathcal{O}_{\mathrm{qc}}$ and $\epsilon>0$, there is an open neighbourhood $U \subset \mathcal{O}_{\mathrm{qc}}$ of $f$ such that for any $g \in U$ and $y \in \partial \mathbb{D},|g(y)-f(y)|<\epsilon$.

Proof. Point evaluation is continuous for $z \in \mathbb{D}$ since convergence in $\mathcal{O}_{\mathrm{qc}}$ implies uniform convergence on compact subsets of $\mathbb{D}$. The continuity of point evaluation on $\partial \mathbb{D}$ follows from the second claim of the Corollary, which we now prove.

Fix $f \in \mathcal{O}_{\mathrm{qc}}$. By Proposition 3.2 and the second claim of Theorem 3.3, there is a $\delta_{1}<0$ and $M>0$ such that if $d_{o}(f, g)<\delta_{1}$ then $|g(y)| /\left|g^{\prime}(0)\right| \leq M$ for all $y \in \partial \mathbb{D}$. Applying Proposition 3.2 and Theorem 3.3, there is a $\delta_{2}$ such that $d_{o}(f, g)<\delta_{2}$ implies that

$$
\left|\frac{g(y)}{g^{\prime}(0)}-\frac{f(y)}{f^{\prime}(0)}\right|<\frac{\epsilon}{2\left|f^{\prime}(0)\right|}
$$


for all $y \in \partial \mathbb{D}$. Since $\chi$ is continuous in its second component there is a $\delta_{3}$ such that $d_{o}(f, g)<\delta_{3}$ implies that

$$
\left|\frac{g^{\prime}(0)}{f^{\prime}(0)}-1\right|<\frac{\epsilon}{2 M\left|f^{\prime}(0)\right|}
$$

Choosing $\delta$ to be the minimum of $\delta_{1}, \delta_{2}$ and $\delta_{3}$, the claim follows from the estimate

$$
\begin{aligned}
|g(y)-f(y)| & =\left|f^{\prime}(0)\right|\left|\frac{g^{\prime}(0)}{f^{\prime}(0)} \frac{g(y)}{g^{\prime}(0)}-\frac{g(y)}{g^{\prime}(0)}+\frac{g(y)}{g^{\prime}(0)}-\frac{f(y)}{f^{\prime}(0)}\right| \\
& \leq\left|f^{\prime}(0)\right|\left(\left|\frac{g^{\prime}(0)}{f^{\prime}(0)}-1\right|\left|\frac{g(y)}{g^{\prime}(0)}\right|+\left|\frac{g(y)}{g^{\prime}(0)}-\frac{f(y)}{f^{\prime}(0)}\right|\right) .
\end{aligned}
$$

Corollary 3.4 immediately implies:

Corollary 3.5. Let $f \in \mathcal{O}_{\mathrm{qc}}$ and $B$ be an open subset of $\overline{\mathbb{C}}$ containing $f(\overline{\mathbb{D}})$. There exists an open neighbourhood $U$ of $f$ in $\mathcal{O}_{\mathrm{qc}}$ such that $g(\overline{\mathbb{D}}) \subset B$ for all $g \in U$.

3.2. Complex structure on $\mathcal{O}_{\mathrm{qc}}(\Sigma)$. In this section we construct a complex Banach manifold structure on $\mathcal{O}_{\mathrm{qc}}(\Sigma)$.

First we outline some facts regarding infinite-dimensional holomorphy (see for example [2], [5, V.5.1] or [8, Section 1.6]). Let $E$ and $F$ be Banach spaces. Let $U$ be an open subset of $E$.

Definition 3.6. A map $f: U \rightarrow F$ is holomorphic if for each $x_{0} \in U$ there is a continuous complex linear map $D f\left(x_{0}\right): E \rightarrow F$ such that

$$
\lim _{h \rightarrow 0} \frac{\left\|f\left(x_{0}+h\right)-f\left(x_{0}\right)-D f\left(x_{0}\right)(h)\right\|_{F}}{\|h\|_{E}}=0 .
$$

Definition 3.7. A map $f: U \rightarrow F$ is called Gâteaux holomorphic if $f$ is holomorphic on affine lines. That is, if for all $a \in U$ and all $x \in E$, the map $z \mapsto f(a+z x)$ is holomorphic on $\{z \in \mathbb{C} \mid a+z x \in U\}$.

Theorem 3.8 ([2, p 198]). Let $f: U \rightarrow F$. The following are equivalent.

(1) $f$ is holomorphic.

(2) $f$ is Gâteaux-holomorphic and continuous.

(3) $f$ is Gâteaux-holomorphic and locally bounded on $U$.

Remark 3.9. In [5, Lemma 5.1 (i) p 206 ] it is stated that Gâteaux holomorphy is sufficient for holomorphy. In fact some further condition such as continuity or local boundedness is necessary.

In order to construct the complex structure on $\mathcal{O}_{\mathrm{qc}}(\Sigma)$, we will need the following Lemma, which says that composition on the left by a holomorphic map is a holomorphic operation on small neighbourhoods in $\mathcal{O}_{q c}$.

Lemma 3.10. Let $K$ be a compact set which is the closure of an open neighbourhood of 0 in $\mathbb{C}$, and let $A$ be an open set containing $K$. Let $U$ be an open set in $\mathcal{O}_{\mathrm{qc}}$ which satisfies 
$\psi(\overline{\mathbb{D}}) \subset K$ for all $\psi \in U$. Let $h: A \rightarrow \mathbb{C}$ be a one-to-one holomorphic map satisfying $h(0)=0$. The map

$$
\begin{aligned}
L_{h}: U & \rightarrow \mathcal{O}_{\mathrm{qc}} \\
f & \mapsto h \circ f
\end{aligned}
$$

is a holomorphic map from $U$ into $\mathcal{O}_{\mathrm{qc}}$.

The existence of a set $U$ satisfying the hypotheses of the Lemma is established by Corollary 3.5. We now proceed with the proof.

Proof. Given the complex structure on $\mathcal{O}_{\text {qc }}$ provided by Theorem 3.1, to show $L_{h}$ is holomorphic we must show that $\chi \circ L_{h} \circ \chi^{-1}: \chi(U) \rightarrow A_{\infty}^{1} \oplus \mathbb{C}$ is holomorphic. That is, $\left(\mathcal{A}(f), f^{\prime}(0)\right) \mapsto\left(\mathcal{A}(h \circ f),(h \circ f)^{\prime}(0)\right)$ is holomorphic. We prove separate holomorphicity and apply Hartogs' theorem (a suitable statement in infinite dimensions can be found in [7]).

It is clear that the map $f^{\prime}(0) \mapsto(h \circ f)^{\prime}(0)=h^{\prime}(0) f^{\prime}(0)$ is holomorphic and continuous, so it remains to be proven that

$$
\mathcal{A}(f) \mapsto \mathcal{A}(h \circ f)
$$

is holomorphic in $A_{\infty}^{1}(\mathbb{D})$. The map is locally (in fact globally) bounded, since $\mathcal{A}\left(\mathcal{O}_{\text {qc }}\right)$ is a bounded subset of $A_{\infty}^{1}(\mathbb{D})$ by the elementary inequality

$$
\left(1-|z|^{2}\right)\left|\frac{f^{\prime \prime}(z)}{f^{\prime}(z)}\right| \leq 6
$$

for univalent functions $f$ satisfying $f(0)=0$. Thus by Theorem 3.8 we need only show that this map is Gâteaux holomorphic.

Choose $f_{0} \in U$. We need to show that $t \mapsto \mathcal{A}\left(h \circ f_{t}\right)$ is a holomorphic curve in $A_{\infty}^{1}(\mathbb{D})$ for complex curves $f_{t} \in \mathcal{O}_{\mathrm{qc}}$ of the following form: $f_{t}$ is the inverse image under $\chi$ of $\left(\mathcal{A}\left(f_{0}\right)+t \phi, q(t)\right)$ where $\phi \in A_{\infty}^{1}(\mathbb{D})$ and $q$ is holomorphic in $t$ with $q(0)=f_{0}^{\prime}(0)$. Since $\chi$ is continuous and $\chi\left(\mathcal{O}_{\mathrm{qc}}\right)$ is open by Theorem 3.1, it follows that $f_{t} \in U$ for $t$ in a small enough neighbourhood of 0 . The explicit solution to this differential equation can be obtained in terms of $\phi, f_{0}$ and $q(t)$ :

$$
f_{t}(z)=\frac{q(t)}{f_{0}^{\prime}(0)} \int_{0}^{z} f_{0}^{\prime}(u) \exp \left(t \int_{0}^{u} \phi(w) d w\right) d u .
$$

Note that for fixed $z, f_{t}(z)$ is holomorphic in $t$. Denoting differentiation with respect to $t$ by $\dot{f}_{t}$, this expression also implies that $\dot{f}_{t}, \dot{f}_{t}^{\prime}, \ddot{f}_{t}$ and $\ddot{f}_{t}^{\prime}$ exist and are holomorphic in $z$.

Let $f_{0} \in U$. Assume that we have a curve $f_{t}$ of the above form for $t \in B$, where $B \subset \mathbb{C}$ is some open neighbourhood of 0 . Shrinking $B$ if necessary, Theorem 3.3 guarantees that $f_{t}(\overline{\mathbb{D}}) \subset K$ for all $t \in B$; that is, $f_{t} \in U$. Define

$$
\alpha(t)=\frac{h^{\prime \prime}}{h^{\prime}} \circ f_{t} \cdot f_{t}^{\prime}
$$

Denoting $t$-differentiation with a dot we then have that

$$
\lim _{t \rightarrow 0} \frac{1}{t}\left(\mathcal{A}\left(h \circ f_{t}\right)-\mathcal{A}\left(h \circ f_{0}\right)\right)=\dot{\alpha}(t)+\phi
$$


where

$$
\dot{\alpha}(t)=\left(\frac{h^{\prime \prime \prime}}{h^{\prime}}-\left(\frac{h^{\prime \prime}}{h^{\prime}}\right)^{2}\right) \circ f_{t} \cdot \dot{f}_{t} f_{t}^{\prime}+\frac{h^{\prime \prime}}{h^{\prime}} \circ f_{t} \cdot \dot{f}_{t}^{\prime} .
$$

It is easy to compute that

$$
\left\|\frac{1}{t}\left(\mathcal{A}\left(h \circ f_{t}\right)-\mathcal{A}\left(h \circ f_{0}\right)\right)-(\dot{\alpha}(t)+\phi)\right\|_{1, \infty}=\left\|\frac{1}{t}(\alpha(t)-\alpha(0)-t \dot{\alpha}(t))\right\|_{1, \infty},
$$

since $\mathcal{A}\left(f_{t}\right)-\mathcal{A}\left(f_{0}\right)=t \phi$. We will show that the right hand side goes to 0 as $t \rightarrow 0$.

By an elementary estimate

$$
|\alpha(t)-\alpha(0)-t \dot{\alpha}(t)| \leq \sup _{|s| \leq|t|}|\ddot{\alpha}(s)| t^{2},
$$

so it only need be shown that $\ddot{\alpha}(t) \in A_{\infty}^{1}(\mathbb{D})$ for $t$ in some neighbourhood of $0 \in \mathbb{C}$. It is easily computed that

$$
\begin{aligned}
\ddot{\alpha}(t)= & \left(\frac{h^{\prime \prime \prime \prime}}{h^{\prime}}-3 \frac{h^{\prime \prime \prime} h^{\prime \prime}}{h^{\prime 2}}-\frac{h^{\prime \prime 3}}{h^{3}}\right) \circ f_{t} \cdot \dot{f}_{t}^{2} f_{t}^{\prime}+\left(\frac{h^{\prime \prime \prime}}{h^{\prime}}-\frac{h^{\prime 2}}{h^{2}}\right) \circ f_{t} \cdot\left(\ddot{f}_{t} f_{t}^{\prime}+2 \dot{f}_{t} \dot{f}_{t}^{\prime}\right) \\
& +\frac{h^{\prime \prime}}{h^{\prime}} \circ f_{t} \cdot \ddot{f}_{t}^{\prime}
\end{aligned}
$$

We claim that there exists an $r>0$ and positive constants $M_{1}, M_{2}, M_{3}$ which bound $f_{s}(z), \dot{f}_{s}(z)$ and $\ddot{f}_{s}(z)$ respectively for all $z \in \mathbb{D}$ and $|s|<r$. We know by Theorem 3.3 that there is a constant $M_{1}$ and $r_{1}>0$ such that $\left|f_{s}(z)\right| \leq M_{1}$ for $|s|<r_{1}$ and $z \in \mathbb{D}$. Let $r_{2}$ and $r^{\prime}$ satisfy $0<r_{2}<r^{\prime}<r_{1}$. Applying Cauchy estimates in the variable $s$ using the curve $|s|=r^{\prime}$ we have that $\left|\dot{f}_{s}(z)\right| \leq r^{\prime}\left|f_{s}(z)\right| /\left|r_{1}-r^{\prime}\right|^{2} \leq M_{2}$ for some $M_{2}$ which is independent of $z \in \mathbb{D}$ and $|s|<r_{2}$. The same argument again shows that there is an $r>0$ and an $M_{3}$ such that $\left|\ddot{f}_{s}(z)\right| \leq M_{3}$ for $|s|<r$ uniformly in $z$, so the claim follows.

Applying the Schwarz lemma to $f_{t}$, we have that $\left(1-|z|^{2}\right)\left|f_{t}^{\prime}(z)\right| \leq\left(M_{1}^{2}-|f(z)|^{2}\right) / M_{1} \leq M_{1}$ for $|t|<r$, and similarly $\left(1-|z|^{2}\right)\left|\dot{f}_{t}^{\prime}(z)\right| \leq M_{2}$ and $\left(1-|z|^{2}\right)\left|\ddot{f}_{t}^{\prime}(z)\right| \leq M_{3}$ for all $|t|<r$ and $z \in \mathbb{D}$. Since $h$ is holomorphic on $K$ and $h^{\prime} \neq 0$, the factors $\left(h^{\prime \prime} / h^{\prime}\right) \circ f_{t}$ etc. of each term in equation (3.2) are bounded by constants $C_{1}, C_{2}$ and $C_{3}$ respectively, uniformly for $z \in \mathbb{D}$ and $|t|<r$. Applying these estimates to equation (3.2) we have

$$
\left(1-|z|^{2}\right)|\ddot{\alpha}(s)(z)| \leq C_{1} M_{1}^{2} M_{2}+C_{2}\left(M_{3} M_{1}+2 M_{2}^{2}\right)+C_{3} M_{3}
$$

which completes the proof.

We may now state and prove the main theorem.

Theorem 3.11. Let $\Sigma$ be a Riemann surface with $n$ distinguished points $p_{1}, \ldots, p_{n}$. The space $\mathcal{O}_{\mathrm{qc}}(\Sigma)$ has a complex Banach manifold structure modelled on $\mathcal{O}_{\mathrm{qc}}^{n}=\mathcal{O}_{\mathrm{qc}} \times \cdots \times \mathcal{O}_{\mathrm{qc}}$.

Proof. Fix a point $\left(\phi_{1}, \ldots, \phi_{n}\right) \in \mathcal{O}_{\mathrm{qc}}(\Sigma)$. We will construct an open neighbourhood and a chart in $\mathcal{O}_{q c}(\Sigma)$.

For $i=1, \ldots, n$, let $D_{i}=\phi_{i}(\mathbb{D})$ be open sets in $\Sigma$. Choose domains $B_{i} \subset \Sigma$ with the following properties: 1) $\phi_{i}(\overline{\mathbb{D}}) \subset B_{i}$, 2) $B_{i} \cap B_{j}=\emptyset$ for $i \neq j$ and 3) $B_{i}$ are open and simply connected. Let $\zeta_{i}: B_{i} \rightarrow \mathbb{C}$ be a local biholomorphic parameter such that $\zeta_{i}\left(p_{i}\right)=0$ and $\zeta_{i}\left(B_{i}\right)$ is bounded. We then have that $\zeta_{i} \circ \phi_{i} \in \mathcal{O}_{\mathrm{qc}}$. 
Let $K_{i}$ be a compact set containing $\zeta_{i} \circ \phi_{i}(\overline{\mathbb{D}})$ in its interior, such that $\zeta_{i}^{-1}\left(K_{i}\right) \subset B_{i}$. By Corollary 3.5. for each $i$ there is an open neighbourhood $U_{i}$ of $\zeta_{i} \circ \phi_{i}$ in $\mathcal{O}_{\text {qc }}$ such that $\psi_{i}(\overline{\mathbb{D}}) \subset K_{i}$ for all $\psi_{i} \in U_{i}$, and so $\left(\zeta_{1}^{-1} \circ \psi_{1}, \ldots, \zeta_{n}^{-1} \circ \psi_{n}\right)$ is an element of $\mathcal{O}_{\mathrm{qc}}(\Sigma)$.

Thus, $U_{1} \times \cdots \times U_{n}$ is an open subset of $\mathcal{O}_{\mathrm{qc}} \times \cdots \times \mathcal{O}_{\mathrm{qc}}$ with the product topology. Let

$$
V_{i}=\left\{\zeta_{i}^{-1} \circ \psi_{i} \mid \psi_{i} \in U_{i}\right\}
$$

and

$$
\begin{aligned}
T_{i}: V_{i} & \rightarrow \mathcal{O}_{\mathrm{qc}} \\
g & \mapsto \zeta_{i} \circ g
\end{aligned}
$$

Of course we have that $T_{i}\left(V_{i}\right)=U_{i}$.

Let $\mathcal{O}_{\mathrm{qc}}(\Sigma)$ be endowed with the topology generated by the open sets of the above form. We define the complex structure on $\mathcal{O}_{\mathrm{qc}}(\Sigma)$ as follows. Let $V=V_{1} \times \cdots \times V_{n} \subset \mathcal{O}_{\mathrm{qc}}(\Sigma)$. The charts are ordered pairs $(T, V)$ where $T=T_{1} \times \cdots \times T_{n}: V \rightarrow \mathcal{O}_{\text {qc }}^{n}$. Consider two charts $(T, V)$ and $\left(T^{\prime}, V^{\prime}\right)$ such that $V \cap V^{\prime} \neq \emptyset$. Let $\zeta_{i}$ and $\zeta_{i}^{\prime}, K_{i}$ and $K_{i}^{\prime}$, etc. correspond to the charts $(T, V)$ and $\left(T^{\prime}, V^{\prime}\right)$ respectively. We have that $\zeta_{i}^{\prime} \circ \zeta_{i}^{-1}$ is holomorphic on $K_{i}$, and $T_{i}^{\prime} \circ T_{i}^{-1}\left(\psi_{i}\right)=\zeta_{i}^{\prime} \circ \zeta_{i}^{-1} \circ \psi_{i}$ for any $\psi_{i} \in T_{i}\left(V_{i} \cap V_{i}^{\prime}\right) \subset \mathcal{O}_{\mathrm{qc}}$. By Lemma 3.10 the map $\psi_{i} \mapsto \zeta^{\prime} \circ \zeta^{-1} \circ \psi_{i}$ is holomorphic on $T_{i}\left(V_{i} \cap V_{i}^{\prime}\right)$. Applying the argument to $T_{i} \circ T_{i}^{\prime-1}$ shows that it is a biholomorphism. Thus $T^{\prime} \circ T^{-1}$ is a biholomorphism.

Remark 3.12. The topology on $\mathcal{O}_{\mathrm{qc}}(\Sigma)$ defined in the proof of Theorem 3.11 is Hausdorff. This can be proved with a short direct argument that relies on the following two facts. (1) $\mathcal{O}_{\mathrm{qc}}$ is Hausdorff. (2) If $\left(\phi_{1}, \ldots, \phi_{n}\right)$ and $\left(\phi_{1}^{\prime}, \ldots, \phi_{n}^{\prime}\right)$ are in $\mathcal{O}_{\mathrm{qc}}(\Sigma)$ then biholomorphic parameters $\zeta_{i}=\zeta_{i}^{\prime}$ can be chosen such that the neighbourhoods $B_{i}$ and $B_{i}^{\prime}$ of the images of $\phi_{i}$ and $\phi_{i}^{\prime}$ are contained in the domain of $\zeta_{i}$.

\section{Appendix: Identification of $\mathcal{O}_{\text {qc }}$ With $Q S\left(S^{1}\right) \times \mathbb{R}$}

We describe the relation of $\mathcal{O}_{\mathrm{qc}}$ to $\mathrm{QS}\left(S^{1}\right)$.

In the following, we say that two domains $E, F$ in $\overline{\mathbb{C}}$ are complementary if $F$ is the complement of the closure of $E$. In particular $\mathbb{D}^{*}$ and $\mathbb{D}$ are complementary.

Theorem 4.1 (conformal welding). Let $\gamma \in Q S\left(S^{1}\right)$. There is a unique $f: \mathbb{D} \rightarrow \mathbb{C}$ and $g: \mathbb{D}^{*} \rightarrow \overline{\mathbb{C}}$ which map onto complementary domains satisfying

(1) $f$ and $g$ are one-to-one, holomorphic, and have quasiconformal extensions to $\overline{\mathbb{C}}$

(2) $\gamma=\left.g^{-1} \circ f\right|_{S^{1}}$

(3) $f(0)=0,\left|f^{\prime}(0)\right|=1$

(4) $g(\infty)=\infty, g^{\prime}(\infty)>0$.

Proof. This is a standard result with a different choice of normalization. By [10, Theorem 2.3] there are maps $f$ and $g$ such that (1) and (4) hold but with (2) and (3) replaced by $\left(2^{\prime}\right) \gamma=\left.g^{-1} \circ f\right|_{S^{1}} \bmod \left(S^{1}\right)$ and $\left(3^{\prime}\right) f(0)=0, f^{\prime}(0)=1$. Property $\left(2^{\prime}\right)$ means that there exists some $\alpha \in \operatorname{Möb}\left(S^{1}\right)$ corresponding to a rotation of $S^{1}$ such that $\gamma=\left.\alpha \circ g^{-1} \circ f\right|_{S^{1}}$. Since $f$ and $g$ are unique, $\alpha$ is also uniquely determined. Setting $\tilde{f}=\alpha^{-1} \circ f$ and $\tilde{g}=\alpha^{-1} \circ g \circ \alpha$ we have that $\tilde{f}$ and $\tilde{g}$ are the unique maps satisfying properties (1) to (4).

Corollary 4.2. Let $m \in \mathbb{R}$ and $\gamma \in Q S\left(S^{1}\right)$. There is a unique $f: \mathbb{D} \rightarrow \mathbb{C}$ and $g: \mathbb{D}^{*} \rightarrow \overline{\mathbb{C}}$ mapping onto complementary domains and satisfying 
(1) $f$ and $g$ are one-to-one, holomorphic, and have quasiconformal extensions to $\overline{\mathbb{C}}$

(2) $\gamma=\left.g^{-1} \circ f\right|_{S^{1}}$

(3) $f(0)=0,\left|f^{\prime}(0)\right|=e^{m}$

(4) $g(\infty)=\infty, g^{\prime}(\infty)>0$.

Proof. Apply Theorem 4.1 to get $\tilde{f}$ and $\tilde{g}$ satisfying its conditions. Then $f=e^{m} \tilde{f}$ and $g=e^{m} \tilde{g}$ satisfies the desired conditions.

Corollary 4.2 establishes the existence of the bijection between $Q S\left(S^{1}\right) \times \mathbb{R}$ and $\mathcal{O}_{\text {qc }}$.

$$
\begin{aligned}
\mathcal{V}: \operatorname{QS}\left(S^{1}\right) \times \mathbb{R} & \rightarrow \mathcal{O}_{\mathrm{qc}} \\
(\gamma, m) & \mapsto f
\end{aligned}
$$

The inverse of this mapping is

$$
\begin{aligned}
\mathcal{V}^{-1}(f)= & \left(\left.g^{-1} \circ f\right|_{S^{1}}, \log \left|f^{\prime}(0)\right|\right) . \\
& \text { REFERENCES }
\end{aligned}
$$

[1] Bers, L. Fiber spaces over Teichmüller spaces. Acta Math. 130 (1973), 89-126.

[2] Chae, Soo Bong. Holomorphy and calculus in normed spaces. With an appendix by Angus E. Taylor. Monographs and Textbooks in Pure and Applied Mathematics, 92. Marcel Dekker, Inc., New York, 1985.

[3] Forstnerič, Franc: Manifolds of holomorphic mappings from strongly pseudoconvex domains. Asian J. Math. 11 (2007) no. 1, 113-126.

[4] Grinshpan, Arcadii Z., Logarithmic geometry, exponentiation, and coefficient bounds in the theory of univalent functions and nonoverlapping domains, in Handbook of complex analysis: geometric function theory vol I., Kühnau, R. ed. Elsevier 2002.

[5] Lehto, Olli. Univalent functions and Teichmüller spaces. Graduate Texts in Mathematics 109 SpringerVerlag, New York, 1987.

[6] Lehto, O. and Virtanen, K. I. Quasiconformal mappings in the plane. 2nd edition. Die Grundlehren der mathematischen Wissenshcaften, Band 126. Springer-Verlag, New York-Heidelberg, 1973.

[7] Mujica, J. Complex Analysis in Banach Spaces, North Holland,Amsterdam, 1986.

[8] Nag, Subhashis. The complex analytic theory of Teichmüller spaces. Canadian Mathematical Society Series of Monographs and Advanced Texts. A Wiley-Interscience Publication. John Wiley \& Sons, Inc., New York, 1988.

[9] Radnell, D. and Schippers, E. Quasisymmetric Sewing in Rigged Teichmueller Space, Commun. Contemp. Math. 8 (2006) no 4, 481-534. arXiv:math-ph/0507031.

[10] Teo, Lee-Peng. Velling-Kirillov metric on the universal Teichmüller curve. J. Anal. Math. 93 (2004), 271-307. arXiv:math.CV/0206202

[11] Takhtajan, Leon A. and Teo, Lee-Peng. Weil-Petersson metric on the universal Teichmüller space. Mem. Amer. Math. Soc., (2006).

Department of Mathematics and Statistics, American University of Sharjah, PO Box 26666, SharJah, UAE

E-mail address, D. Radnell: dradnell@aus.edu

Department of Mathematics, University of Manitoba, Winnipeg, MB, R3T 2N2, Canada

E-mail address, E. Schippers: eric_schippers@umanitoba.ca 